\title{
Virtual Screening and Biomolecular Interactions of CviR-Based Quorum Sensing Inhibitors Against Chromobacterium violaceum
}

\author{
Vinothkannan Ravichandran ${ }^{\dagger}$, Lin Zhong ${ }^{\dagger}$, Hailong Wang, Guangle Yu, Youming Zhang * \\ and Aiying $\mathrm{Li}^{*}$ \\ State Key Laboratory of Microbial Technology, Shandong University-Helmholtz Institute of Biotechnology (SHIB), School of \\ Life Science, Shandong University, Qingdao, China
}

\section{OPEN ACCESS}

Edited by:

Rodolfo García-Contreras, Universidad Nacional Autónoma de México, Mexico

Reviewed by: John Pinney,

Imperial College London, United Kingdom

Yael González Tinoco,

Universidad Nacional Autónoma de México, Mexico

${ }^{*}$ Correspondence: Youming Zhang zhangyouming@sdu.edu.cn

Aiying $\mathrm{Li}$

ayli@sdu.edu.cn

these authors have contributed equally to this work

Received: 04 May 2018

Accepted: 30 July 2018

Published: 04 September 2018

Citation:

Ravichandran V, Zhong L, Wang $H$, Yu G, Zhang Y and Li A (2018) Virtual

Screening and Biomolecular Interactions of CviR-Based Quorum

Sensing Inhibitors Against

Chromobacterium violaceum. Front. Cell. Infect. Microbiol. 8:292.

doi: 10.3389/fcimb.2018.00292
The rise of bacterial multi drug resistance becomes a global threat to the mankind. Therefore it is essential to find out alternate strategies to fight against these "super bugs." Quorum sensing (QS) is a cell-to-cell communication mechanism by which many bacteria regulate their biofilm and virulence factors expression to execute their pathogenesis. Hence, interfering the quorum sensing is an effective alternate strategy against various pathogens. In this study, we aimed to find out potential CviR-mediated quorum sensing inhibitors (QSIs) against Chromobacterium violaceum. Virtual screening from a natural products database, in vitro biofilm and violacein inhibition assays have been performed. Biofilm formation was investigated using confocal microscopy and gene expression studies were carried out using qRT-PCR. Further, to study the biomolecular interaction of QSIs with purified CviR Protein (a LuxR homologue), microscale thermophoresis (MST) analysis was performed. Results suggested that phytochemicals SPL, BN1, BN2, and C7X have potential GScore when compared to cognate ligand and reduced the biofilm formation and violacein production significantly. Especially, $100 \mu \mathrm{M}$ of BN1 drastically reduced the biofilm formation about $82.61 \%$. qRT-PCR studies revealed that cvil, cviR, vioB, vioC, vioD genes were significantly down regulated by QSIs. MST analysis confirmed the molecular interactions between QSIs and purified CviR protein which cohere with the docking results. Interestingly, we found that BN2 has better interaction with CviR $\left(K_{d}=45.07 \pm 1.90 \mathrm{~nm}\right)$. Overall results suggested that QSIs can potentially interact with CviR and inhibit the QS in a dose dependent manner. Since, LuxR homologs present in more than 100 bacterial species, these QSIs may be developed as broad spectrum anti-infective drugs in future.

Keywords: Chromobacterium violaceum, quorum sensing, quorum sensing inhibition, virtual screening, biofilm inhibition and microscale thermophoresis

\section{INTRODUCTION}

Antibiotic resistance has become a global health issue and considered to be a leading health challenge in recent years (Ferri et al., 2017). Hence, efforts have to be taken to identify novel strategies which could curb bacterial pathogenesis in order to tackle multi drug resistant (MDR) "super bugs" (Wagner et al., 2016). Bacteria coordinates their behavior through quorum sensing 
(QS), a mechanism that helps bacterial populations to enable harmonious responses including biofilm formation and virulence factors expressions. Since, QS regulates the virulence arsenal of many pathogenic bacteria, it seems to be a captivating drug target to combat bacterial infections (Rasmussen and Givskov, 2006; Williams, 2017). Drugs targeting the virulence pathways could curb the bacterial pathogenesis and thereby prevents the disease development.

$\mathrm{N}$-acylhomoserine lactones (AHLs) and peptides are the autoinducers in gram-negative bacteria and gram-positive bacteria respectively. Furthermore, autoinducer-2 (AI-2) are reported as interspecies communication signal (Miller and Bassler, 2001). AHLs contains a homoserine lactone ring with varying length of acyl chains (C4 to $\mathrm{C} 18$ ) via amide bonds (Bassler, 2002). In LuxI/LuxR- based QS systems, AHLs are synthesized by LuxI synthases and LuxR encodes the receptor proteins. Once synthesized, AHLs will be internalized, accumulated and recognized by LuxR-type receptor and this will modulate the regulation of target genes (Paul et al., 2017).

Chromobacterium violaceum, a gram-negative, facultative anaerobic, non-sporing coccobacillus has a quorum-sensing system consists of CviI/CviR, a LuxI/LuxR homolog (McClean et al., 1997; Stauff and Bassler, 2011). It is demonstrated that inhibitors able to interact with CviR could prevent the nematode from C. violaceum-mediated killing. Hence, it is apparent that the quorum sensing plays a vital role in C. violaceum pathogenesis and it is established that QSIs could be potent drug candidates in the battle against MDR pathogens including C. violaceum (Swem et al., 2009; Chen et al., 2011).

The advantages of QSIs over conventional antibiotics are as follows. Firstly, it is believed that the pathogens would not develop resistance to QSIs as this strategy may create only no or little selective pressure to the bacteria (Defoirdt et al., 2010). Secondly, QS seems to be essential for spreading bacterial resistance as it is directly or indirectly influencing the horizontal gene transfer. Thirdly, the LuxI/LuxR homologs have been reported in more than 100 Gram-negative bacterial species and over 200 different Gram-negative bacteria have been described to use AHLs as QS signals. Thereby QSIs may have the competence to be a broad range anti-virulent drugs (Adonizio et al., 2006). Taken together, interfering this mechanism would have an astounding impact over the bacterial resistance and its control (Uroz et al., 2009; Kalia, 2015). Numerous studies have been published related to quorum sensing inhibitors (QSIs) which rationalize the capability of this strategy (Ren et al., 2005; Rasmussen and Givskov, 2006; Ni et al., 2008; Kalia, 2013; Brackman and Coenye, 2015; Coughlan et al., 2016; Delago et al., 2016).

Natural products have always been fascinating source for the drug discovery. It is reported that more than $80 \%$ of drugs were natural products or inspired by a natural compound (Harvey, 2008). It is evident that almost half of the drugs approved in last two decades are based on natural products (Butler, 2008). Hence, it is crucial to screen natural products to discover potential QSIs against MDR pathogens. Despite the fact, several studies revealed that numerous plant extracts and natural products inhibit the quorum sensing of various pathogens (Adonizio et al., 2006; Vattem et al., 2007; Bouyahya et al., 2017; Paul et al., 2017), in-depth investigations are much essential to take-up these QSIs to the next level of drug discovery.

Here, we report high-throughput virtual screening of QSIs against CviR, the quorum regulator of $C$. violaceum and their biological evaluation through in vitro assays including qRT-PCR. To the best of our knowledge, this is the first study to discuss the molecular interactions of QSIs with purified quorum sensing target protein, CviR using microscale thermophoresis (MST) analysis.

\section{MATERIALS AND METHODS}

\section{Bacterial Strains and Growth Conditions}

C. violaceum 31532, E.coli BL21 (DE3) were used in this study. All the bacterial strains were grown in Luria-Bertani (LB) medium, and C. violaceum 31532 and E.coli BL21 (DE3) were grown at $30^{\circ}$ and $37^{\circ} \mathrm{C}$ respectively, for $24 \mathrm{~h}$. Quorum sensing inhibitors (QSIs) Sappanol (SPL), Butein (BN1), Bavachin (BN2), and Catechin 7-xyloside (C7X) were purchased from Chemfaces, China.

\section{High Throughput Virtual Screening (HTVS)}

The virtual screening was performed against CviR using Schrodinger software (Maestro v10.6, Glide module) to screen the natural product database containing 4687 compounds. The energy minimized 3D ligand file was prepared using LigPrep module (Friesner et al., 2006). The three-dimensional structure of CviR protein was retrieved from Protein Data Bank (PDB: 3QP1 and 3QP5). Coordinates of CviR structure was prepared by using protein preparation wizard. Docking was performed using GLIDE (Grid Based Ligand Docking with Energetics) module in Schrodinger suite. Grid files were generated using the $\mathrm{C}_{6} \mathrm{HSL}$, the native ligand $\left(\mathrm{C}_{6} \mathrm{HSL}\right)$ to the center of both the grid boxes. Tyr 80, Trp 84, Asp 97, and Ser 155 were found to be the active site residues. The compounds were subjected to HTVS ligand docking using the pre-computed grid files and then XP docking was also performed for top ranking compounds. The XP docking helps to remove the false positives with much stricter scoring function than the HTVS. Hits having least GScore (Glide score) and more number of $\mathrm{H}$-bonds were analyzed further. To investigate the binding pocket of LuxR homologs, CviR from C. violaceum and LasR from Pseudomonas aeruginosa were compared using RCSB PDB Protein Comparison Tool.

\section{Biofilm Inhibition Assay}

The effect of QSIs on biofilm formation was measured by microtitre plate assay (O'toole and Kolter, 1998). Briefly, overnight cultures $(0.4 \mathrm{OD}$ at $600 \mathrm{~nm})$ of C.violaceum were added into $1 \mathrm{~mL}$ of fresh LB medium and grown with or without QSIs with varying concentrations (1, 10 and $100 \mu \mathrm{M})$ for $24 \mathrm{~h}$ at $30^{\circ} \mathrm{C}$. After incubation, microtitre plates were washed with PBS $\left({ }_{\mathrm{p}} \mathrm{H}\right.$ 7.4) to remove the free-floating planktonic cells. The biofilm was stained using $200 \mu \mathrm{L}$ of $0.1 \%$ crystal violet (CV) solution. After $15 \mathrm{~min}, \mathrm{CV}$ 
solution was removed and $200 \mu \mathrm{L}$ of $95 \%$ ethanol was added. The biofilm was then quantified by measuring the absorbance at OD $470 \mathrm{~nm}$ using microplate reader (Infinite M200, Tecan).

\section{Violacein Quantification Assay}

Production of violacein pigment by C. violaceum in the presence and absence of QSIs was analyzed by violacein extraction and quantification (Blosser and Gray, 2000). Briefly, overnight culture $\left(\mathrm{OD}_{600} \mathrm{~nm}=0.1\right)$ was incubated in conical flask containing LB broth with or without QSIs $(1,10$, and $100 \mu \mathrm{M})$ and incubated at $30^{\circ} \mathrm{C}$ for $24 \mathrm{~h}$. Bacterial cells were then collected and the pellet was dissolved in $1 \mathrm{~mL}$ DMSO. Cell debris was removed by centrifugation at $13,000 \mathrm{~g}$ for $10 \mathrm{~min}$ and the absorbance of soluble violacein was read at $585 \mathrm{~nm}$ using microplate reader (Infinite M200, Tecan).

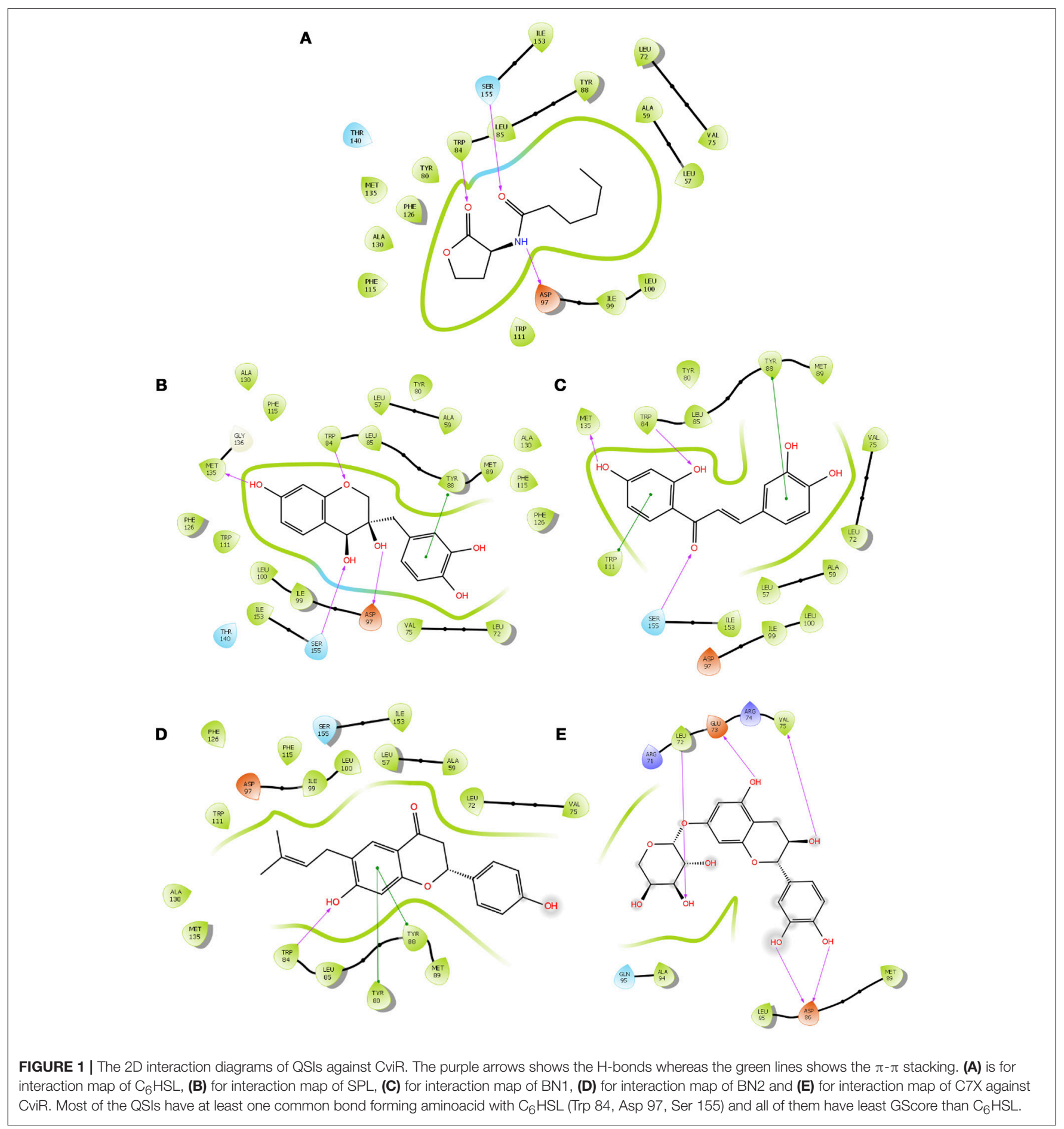




\section{Confocal Laser Scanning Microscopy (CLSM) Studies}

Confocal Laser Scanning Microscopy (CLSM) analysis of the C.violaceum biofilms was performed as described by (Zhao and Liu, 2010). Static biofilms were grown on a glass cover slips (1: 100 diluted culture of C.violaceum inoculated in LB broth and incubated overnight at $30^{\circ} \mathrm{C}$ in stationary condition) in 6well cell culture plates either with or without QSIs $(100 \mu \mathrm{M})$. The developed biofilms were washed twice to remove loosely bound cells and stained with FITC-ConA for $15 \mathrm{~min}$. Cells were rinsed twice in PBS to remove the excess stains and the adhered cells were analyzed using CLSM (Zeiss L800, Japan) with the excitation and emission wavelength set at 488 and $520 \mathrm{~nm}$ respectively.

\section{qRT-PCR Studies}

Total RNA was extracted from C. violaceum biofilm cells using the RNAprep Pure Kit (Tiangen, China) as per manufacturer's instructions. Biofilm cells were grown in $1 \mathrm{~mL} \mathrm{LB}$ medium with or without QSIs $(100 \mu \mathrm{M})$ at $30^{\circ} \mathrm{C}$ for $24 \mathrm{~h}$. Total RNA was extracted with the RNA isolation kit (TIANGEN Biotech Co., Ltd., Beijing, China). Primers for $c v i I, c v i R, v i o B$, vioC, vioD, and rpoD (Table S1) were synthesized by Sangon Biotech (Shanghai, China). Total RNA was used as a template for the reverse transcription reaction using a Prime Script RT Reagent
Kit (TaKaRa, Japan) at $37^{\circ} \mathrm{C}$ for $15 \mathrm{~min}$, three times (reverse transcription), and at $85^{\circ} \mathrm{C}$ for $5 \mathrm{~min}$ (reverse transcriptase inactivation) as per the manufacturer's protocol, with a total volume of $20 \mu \mathrm{L}$.

The qRT-PCR was performed according to the manufacturer's protocol for the SYBR ${ }^{\circledR}$ Premix Ex TaqTM II Kit (TaKaRa, Japan). Reverse transcriptase was used as a template for RTqPCR, and the total reaction system $(20 \mu \mathrm{L})$ was made up as the following: $10 \mu \mathrm{L}$ SYBR ${ }^{\circledR}$ Premix Ex TaqTM II $(2 \times), 0.8 \mu \mathrm{L}$ forward primer, $0.8 \mu \mathrm{L}$ reverse primer, $0.4 \mu \mathrm{L}$ ROX Reference Dye $(50 \times), 2 \mu \mathrm{L}$ DNA template, and $6 \mu \mathrm{L}$ double-distilled $\mathrm{H}_{2} \mathrm{O}$ $\left(\mathrm{ddH}_{2} \mathrm{O}\right)$. Afterwards, qRT-PCR was performed using Applied Biosystems Quant Studio ${ }^{\mathrm{TM}} 3$ Real-Time PCR System (Applied Biosystems Inc., CA, USA). The reaction conditions are as follows: pre-denaturation at $95^{\circ} \mathrm{C}$ for $30 \mathrm{~s}$, followed by 40 cycles of denaturation at $95^{\circ} \mathrm{C}$ for $5 \mathrm{~s}$ and annealing and extension at $60^{\circ} \mathrm{C}$ for $30 \mathrm{~s}$. $r p o D$ was used as an internal reference. The relative mRNA expression of all the genes were calculated using the 2$\Delta \mathrm{Ct}$ method. The experiment was independently conducted 3 times.

\section{Expression and Purification of CviR}

The DNA fragment encoding CviR (GQ398094) was amplified using the primers 5'-CGATATTATTGAGGCTCACAGAG AACAGATTGGTGGATCCATGGTGATCTCGAAACCCA

TABLE 1 | Docking analysis of Quorum sensing inhibitors against CivR, the quorum regulator of Chromobacterium violaceum.

\begin{tabular}{|c|c|c|c|c|c|}
\hline S.No & Name & Structure & GScore & Number of $\mathrm{H}$-bonds & $\begin{array}{l}\text { Bond forming } \\
\text { amino acids }\end{array}$ \\
\hline 1 & $\mathrm{C}_{6} \mathrm{HSL}$ & & -7.052 & 3 & $\begin{array}{c}\text { Trp } 84 \\
\text { Asp97 } \\
\text { Ser } 155\end{array}$ \\
\hline 2 & Sappanol & & -12.140 & 4 & $\begin{array}{c}\text { Trp } 84 \\
\text { Asp } 97 \\
\text { Met } 135 \\
\text { Ser } 155\end{array}$ \\
\hline 3 & Butein & & -11.246 & 3 & $\begin{array}{c}\text { Trp } 84 \\
\text { Met } 135 \\
\text { Ser } 155\end{array}$ \\
\hline 4 & Bavachin & & -8.056 & 1 & Trp 84 \\
\hline 5 & Catechin 7-xyloside & & -7.414 & 4 & $\begin{array}{c}\text { Glu73 } \\
\text { Val } 75 \\
\text { Asn } 77 \\
\text { Asp } 86\end{array}$ \\
\hline
\end{tabular}




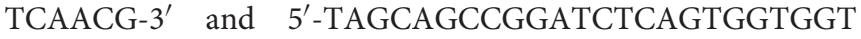
GGTGGTGGTGCTCGAGTTATTCGTTCGCTACGGTCGA G-3'. Primers having homology arm were used for the Red/ET recombination (Wang et al., 2016). PCR products were cloned into the expression plasmid pET28a (Novagen) linearized with BamHI and XhoI restriction enzymes by LLHR method (linear plus linear homologs recombination) of Red/ET in GBdir as per our previous reports (Wang et al., 2016). The resulting plasmid was termed as pET28-CviR and was verified by DNA sequencing of the insert and flanking regions. Produced protein corresponds to a fusion of CviR with the SUMO tag and His-tag as well. E. coli BL21 (DE3) was transformed with pET28-CviR. 5L Erlenmeyer flasks containing 2L LB medium supplemented with $5 \mu \mathrm{g} / \mathrm{ml}$ of kanamycin were inoculated with an overnight culture of E. coli BL21 (DE3) pET28-CviR to an initial OD 660 of 0.05 . Growth was carried out at $37^{\circ} \mathrm{C}$ until the $\mathrm{OD} 660$ of 0.4. The temperature was then lowered to $18^{\circ} \mathrm{C}$ and growth continued until an $\mathrm{OD}_{660}$ of $0.6-0.8$, and then induced with $0.1 \mathrm{mM}$ IPTG and growth was continued overnight at $18^{\circ} \mathrm{C}$. All subsequent manipulations were conducted at $4^{\circ} \mathrm{C}$. The cells were harvested by centrifugation at 4,200 rpm and ultrasonicated for $14 \mathrm{~min}$ in $80 \mathrm{~mL}$ of washing buffer with $14 \%$ of machine power (250 mM Tris- $\mathrm{HCl}, 150 \mathrm{mM} \mathrm{NaCl}, 20 \mathrm{mM}$ imidazole, 5\% glycerol, ${ }_{\mathrm{p}} \mathrm{H}$ 7.8) to break the cell wall for purification. Further, samples were centrifuged at 11,000 rpm for $1 \mathrm{~h}$ and washed twice with washing buffer after flow-through the supernatant through Ni-sepharose 6 Fast flow (GE Healthcare, USA. $20 \mathrm{~mL}$ wash buffer was used to elute the CviR protein once digested with ULP proteinase. The protein was stored at $-80^{\circ} \mathrm{C}$ for further use.
A

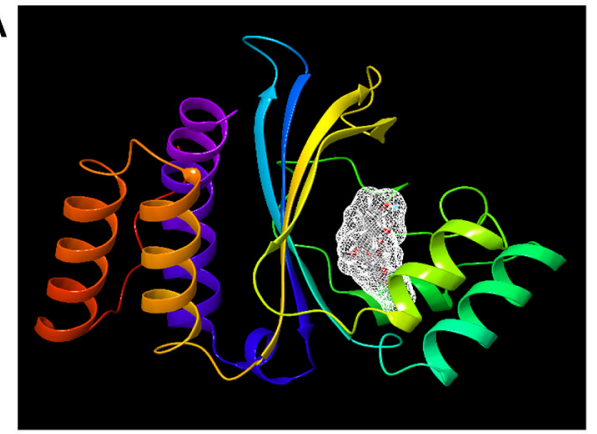

C

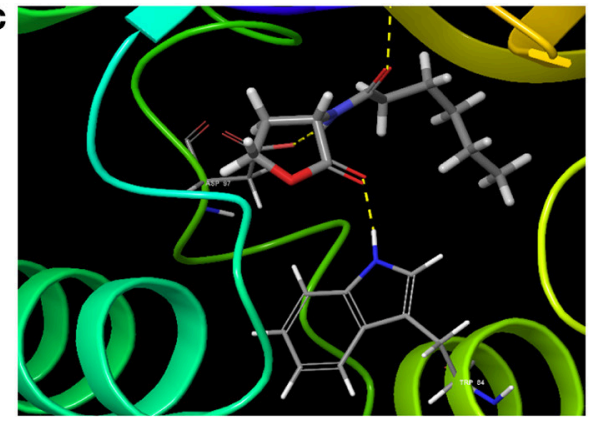

E

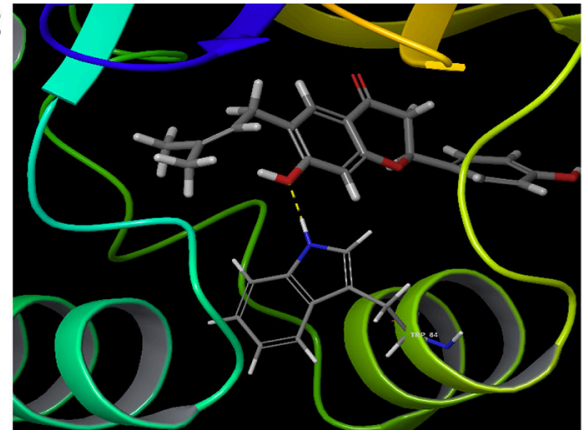

B

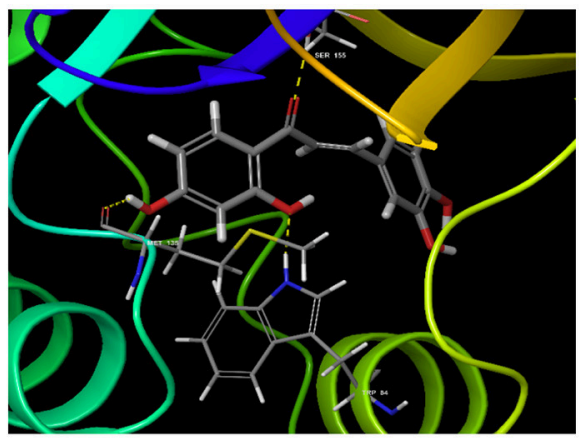

D

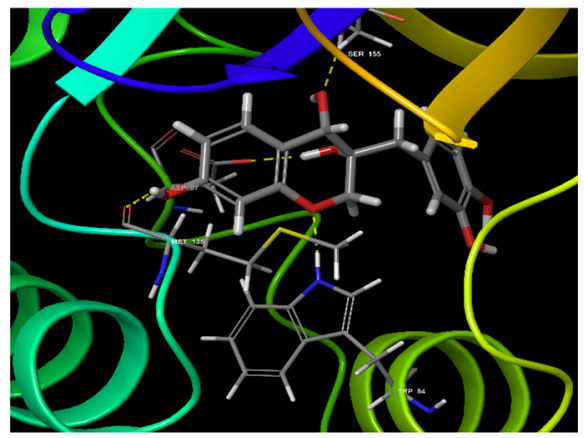

$\mathbf{F}$

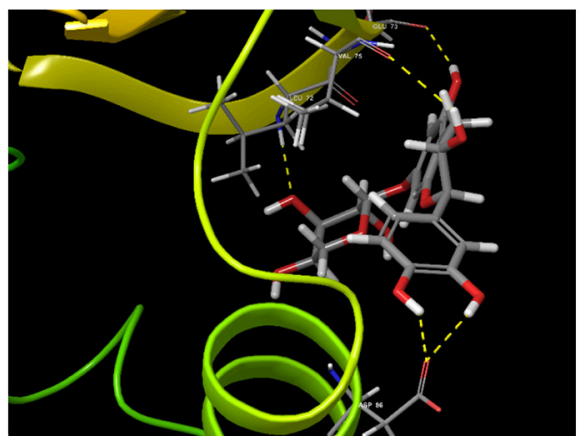

FIGURE 2 | The 3D molecular interactions of QSIs against CviR (A) is for CviR, the LuxR homolog of C. violaceum where the ligand binding pocket is depicted in light gray (B) is for molecular interaction of $\mathrm{C}_{6} \mathrm{HSL}$ with $\mathrm{CviR}$ (3 H bonds) (C) for molecular interaction of SPL (4 H bonds) (D) for molecular interaction of BN1 (3H bonds) (E) for molecular interaction of BN2 (1 H bond) and $\mathbf{( F )}$ for molecular interaction of C7X against CviR (4 H Bonds). $\mathrm{H}$-bonds represented in yellow dotted line. 


\section{MST Analysis}

All the compounds were analyzed with the concentration gradient of $50 \mu \mathrm{M}$ with $20 \mu \mathrm{M}$ of CviR which was labeled Monolith $\mathrm{NT}^{\mathrm{TM}}$ Protein Labeling Kit RED-NHS (Cat Nr: L001) before instrumental analysis. LED power was 20\% and MST optimized buffer was used for the analysis $(50 \mathrm{mM}$ Tris- $\mathrm{HCl}$, $150 \mathrm{mM} \mathrm{NaCl}, 10 \mathrm{mM}$ MgCl2, 0,05\% Tween-20).

Analysis was performed on Monolith NanoTemper (NT) 115 and its accessory, i.e., standard-treated $4 \mu \mathrm{L}$ volume glass capillaries were employed to measure the molecular interaction (NanoTemper Technologies GmbH, Munich, Germany). Means of fluorescence intensity obtained by the MST measurements were fitted and the resultant $K_{\mathrm{d}}$ values were given together with an error estimation from the fit by the built-in formula of NT 1.5.41 analysis software (Cai et al., 2017).

\section{Statistical Analysis}

Graph pad prism software (version 6.01) was used for statistical analysis. One way ANOVA and multiple comparisons were carried out wherever required. $P$-values $(<0.05$ and $<0.01)$ were considered as statistically significant. All the assays were conducted in triplicates and the results were expressed as mean \pm SD.

\section{RESULTS}

\section{Computational Studies}

\section{Molecular Interaction of QSIs Against CviR}

To screen quorum sensing inhibitors against CviR, QS regulator of $C$. violaceum, virtual screening was performed using a natural product database. GScore for the native ligand $\left(\mathrm{C}_{6} \mathrm{HSL}\right)$ was -7.052 and $\mathrm{C}_{6} \mathrm{HSL}$ was able to form three $\mathrm{H}$-bonds with Trp 84, Asp 97, Ser 155 (Figures 1A-E) which was used as reference value and pattern of interaction for the pose analysis. SPL, BN1, BN2, C7X were having the GScore $-12.140,-11.246,-8.056$, -7.414 (Table 1) respectively. SPL was able to form $4 \mathrm{H}$-bonds with amino acids Trp 84, Asp 97, Met 135 and Ser 155 along with a pi-pi stacking with Tyr 88 . BN1 was able to form $3 \mathrm{H}$-bonds

A

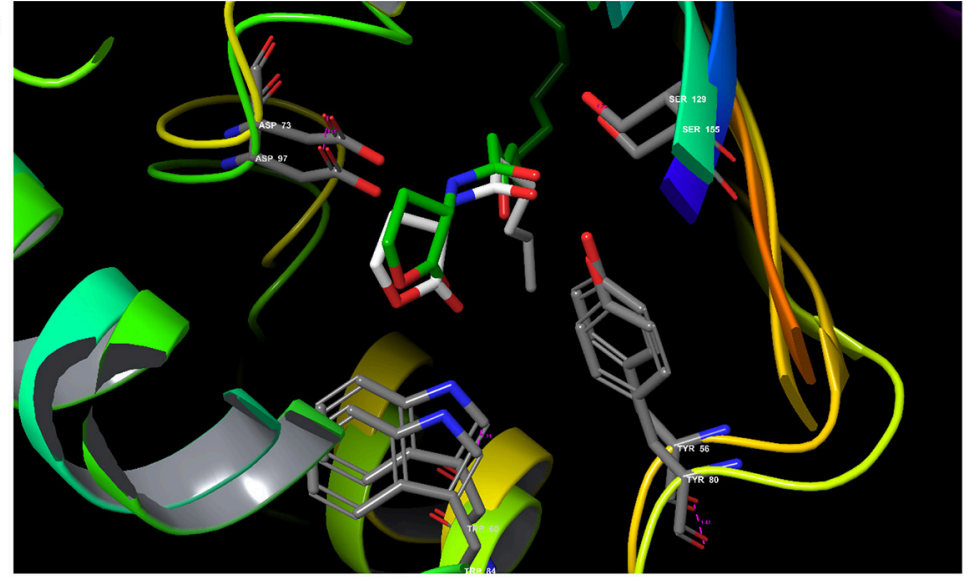

B

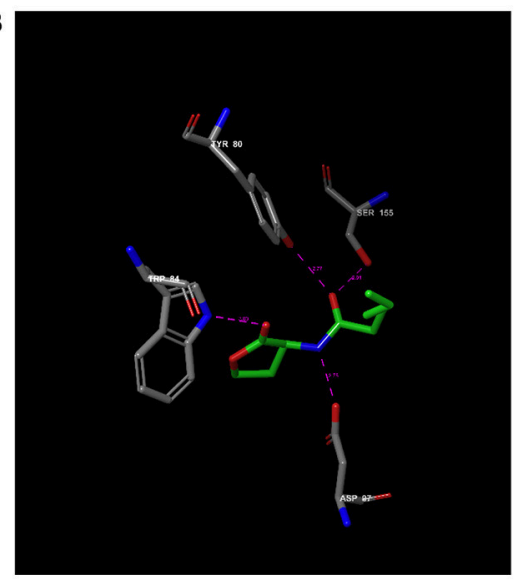

C

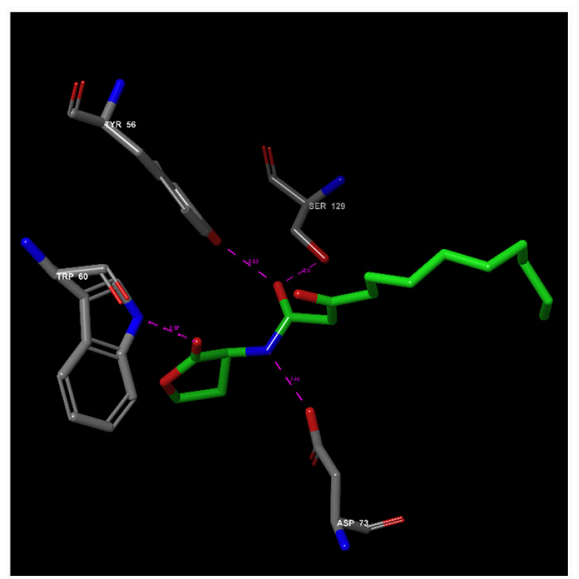

FIGURE 3 | Comparative analysis of CviR with LasR using RCSB PDB Protein Comparison Tool. (A) Molecular comparison of CviR vs LasR. (A) The alignment of the active site residues (Tyr, Trp, Asp, Ser) which are crucial for the interactions. (B) The molecular interaction of (N-hexanoyl-L-Homoserine lactone ( $\left.\mathrm{C}_{6} \mathrm{HSL}\right)$ against CviR (C) The molecular interaction of N-(3-Oxododecanoyl)-L-homoserine lactone (3-Oxo- $\mathrm{C}_{12}$-HSL) with LasR. 
with Trp 84, Met 135 and Ser 155 along with 2 pi-pi stacking with Trp 84 and Tyr 88 . Whereas, BN2 was able to form only one $\mathrm{H}$-bond with two pi-pi interaction with Tyr80 and Tyr 88 (Figures 2A-F). In contrast, C7X has a very unusual pattern of interaction and it was able to form $4 \mathrm{H}$-bonds with Glu73, Val 75, Asn 77, and Asp 86. After pose analysis, based on GScore and $\mathrm{H}$-bond forming ability, the ligands were chosen for further studies.

\section{Comparative Analysis of CviR vs. LasR}

To verify how close the binding pocket LuxR homologs are, the PDB structure of CviR and LasR were analyzed. As expected, both cognate ligands were interacting with the very similar amino acids in both receptors. $\mathrm{C}_{6} \mathrm{HSL}$ was interacting with Tyr 80, Trp 84, Asp 97, and Ser 155 in CviR and 3-oxo-C ${ }_{12}$ HSL was interacting with Tyr 56, Trp 60, Asp 73 and Ser 129 (Figure 3A). Further, positional changes of these residues were calculated and found to have very minute change. The distance between Tyr 80-56 was $1.43 \AA$ and the distance between Trp84-60 was $1.14 \AA$ (Figures 3B,C). Asp 97-73 were in a distance of $1.00 \AA$. Surprisingly, Ser155-129 were in a distance less than $1 \AA(0.75 \AA)$. Further, we found that SPL and BN1 were able to interact with Ser 155, which is crucial for CviR and LasR as well.
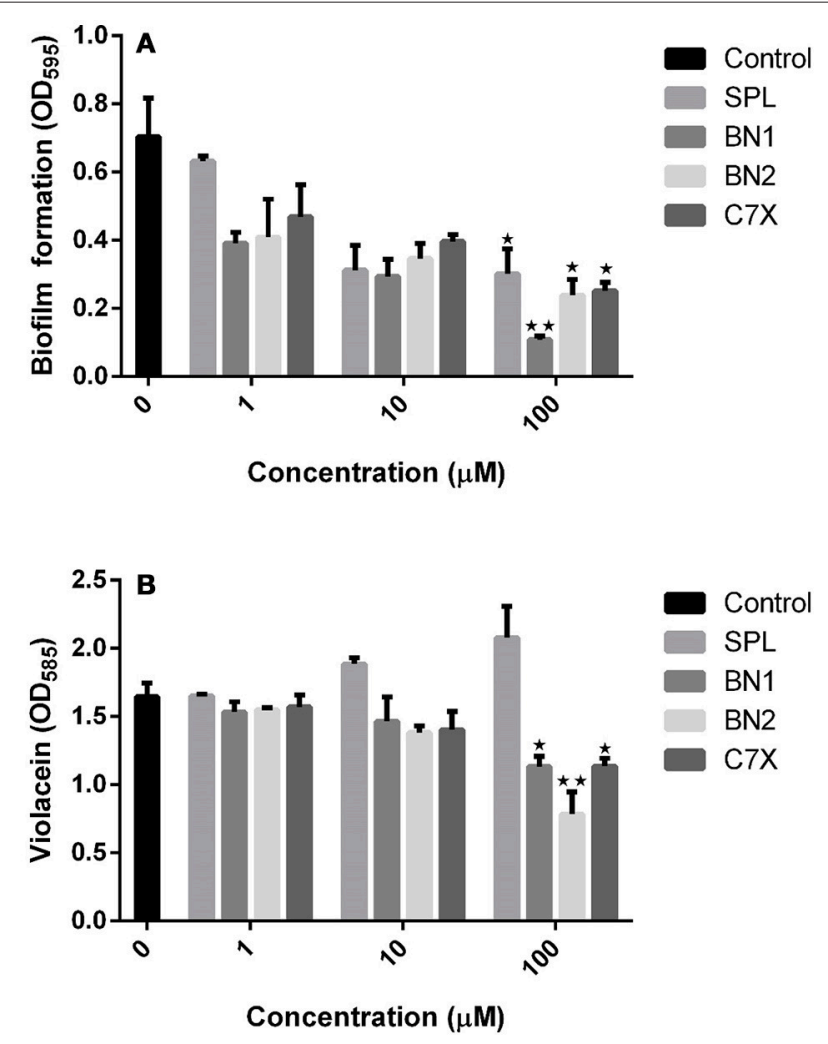

FIGURE 4 | The effect of QSI on biofilm formation and violacein. (A) The biofilm formation was inhibited by QSIs. Especially, BN2 and C7X have reduced the biofilm formation significantly whereas BN1 decreased the biofilm formation drastically ( 50\%). (B) The similar trend was followed in violacein production also. Though all the three QSIs reduce the violacein production, the SPL found be increased. ${ }^{*} P<0.05$ and ${ }^{\star \star} P<0.01$.

\section{Influence of QSIs on Biofilm, Growth, and Violacein}

It is essential to verify the efficacy of QSIs against quorum sending regulated phenotypes in C.violaceum. Biofilm is one of the major factor that is under the control of quorum sensing and plays a crucial role in pathogenesis and drug resistance. All the tested QSIs reduced the biofilm formation significantly at varying concentrations $(1,10$ and $100 \mu \mathrm{M})$. Except C7X, all the QSIs reduced more than 50\% of biofilm at $10 \mu \mathrm{M}$ concentrations (Figure 4A). Especially, BN1 significantly reduced the biofilm formation about $82.61 \%$ when supplied with $100 \mu \mathrm{M}$. Whereas, BN2 and C7X reduced the biofilm by about 66 and $64.26 \%$ respectively with the similar treatment. To differentiate the quorum sensing inhibition activity of these QSIs from antibiotic activity, the growth was analyzed. Except SPL, none of the QSIs found to have influence on the growth of C. violaceum (Figure S1). Violacein, a purple pigment produced by the C. violaceum which is reported to be under the control of QS mechanism via vioABCDE operon. Violacein quantification analysis revealed that all the tested QSIs have potentially suppressed the violacein production. BN2 reduced the violacein drastically by $52.50 \%$ when treated with $100 \mu \mathrm{M}$ (Figure 4B). A concentration-dependent reduction in the violacein production was observed. Unfortunately, SPL was found to increase the production of violacein by 14.51 and $26.26 \%$ when treated with 10 and $100 \mu \mathrm{M}$ respectively.

\section{Confocal Studies}

The efficacy of the QSIs on biofilm development was examined using the confocal laser scanning microscopy (CLSM). It was found that all the QSIs except SPL were negatively regulating the biofilm formation when treated with $100 \mu \mathrm{M}$ concentrations (Figures 5A-E). To be specific, BN2 and BN2 have significantly reduced the biofilm formation when administered with $100 \mu \mathrm{M}$.

\section{Gene Expression Studies}

To investigate the impact of QSIs $(100 \mu \mathrm{M})$ on the genes expression related to C.violaceum quorum sensing, qRT-PCR studies have been performed. First of all, the effect of QSIs on cviI and $c v i R$ was evaluated. Data suggest that the BN1 and C7X were able to significantly suppress the expression of $c$ viI (Figure 6A). Whereas in case of cviR, the similar pattern of decrement was observed which is comparable to that of $c v i I$. It is noteworthy, that SPL increased the expression of $c v i I$ but comparatively less in $c v i R$.

To study further the effect of QSIs on vioABCD operon, genes including vioB, vioC, vioD were analyzed. All the QSIs were significantly reduced the expression of vioB, vioC, vioD genes (Figure 6B). Especially, BN2 decreased the expression of these genes very significantly. Surprisingly, SPL also decreased the expressions.

\section{Expression and Purification of CviR}

RecET based direct cloning and $\operatorname{Red} \alpha \beta$ based recombineering were used for heterologous expression of CviR Protein. The $c v i R$ gene was cloned into the expression vector pET28a and the protein was expressed in E. coli (Figure 7). The purification were 

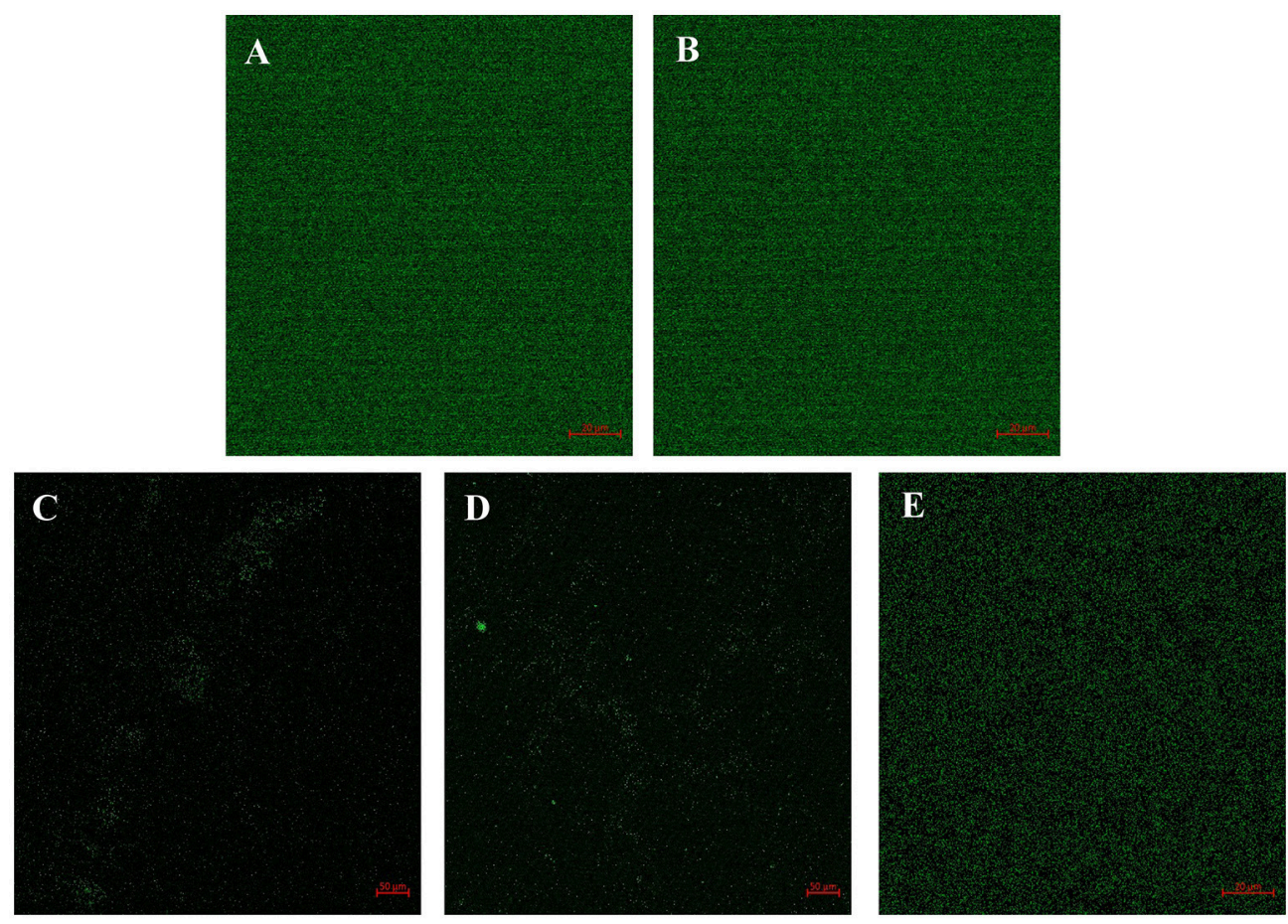

FIGURE 5 | Confocal image shows the efficacy of QSls to curb the biofilm formation. (A) Control (B) SPL (C) BN1 (D) BN2 and (E) C7X.

carried out under different conditions of cell growth and buffers. The purified protein was further investigated by polyacrylamide gel electrophoresis (PAGE) (Figure S2).

\section{Microscale Thermophoresis}

MST experiment was carried out to detect the molecular interaction between QSIs and CviR. Differences in normalized fluorescence of the bound and unbound state will allow determination of the fraction bound and thus the dissociation constant is calculated. All values are multiplied by a factor of 1,000 which yields the relative fluorescence change in per thousand. MST results suggested that all the QSIs except C7X have significant binding ability. It is noteworthy to mention that the dissociation constant $\left(K_{\mathrm{d}}\right)$ of $\mathrm{BN} 2$ is $45.07 \pm 1.90 \mathrm{~nm}$ (Figure 8C). Surprisingly, the $\mathrm{F}_{\text {norm }}$ value of BN1 is increased when the concentration increased and having a sigmoidal curve suggests a competitive interaction pattern (Figure 8B).

\section{DISCUSSION}

Due to misuse and overuse of antibiotics along with complex bacterial drug resistance mechanisms, antimicrobial resistance has emerged as a global threat (Soukarieh et al., 2018). According to WHO's Global Antimicrobial Surveillance System (GLASS), occurrence of MDR infections was found among half a million people all around the world. Resistance to penicillin has raised up to $51 \%$ whereas ciprofloxacin resistance raised from 8 to $65 \%$. The recent report from GLASS confirms that there is a serious situation of antibiotic resistance worldwide (Tornimbene et al., 2018). Many case reports on C.violaceum infections were published with variety of health complications and it is resistant to a broad range of antibiotics including rifampin, vancomycin, ampicilin and cephalosporins (Fantinatti-Garboggini et al., 2004; Justo and Durán, 2017). Hence, alternatives to antibiotics is the "need of the hour" which will ultimately reduce morbidity, mortality and economic burden (Laxminarayan et al., 2016). Recently, disarming the bacterial virulence seems to be a potential alternate strategy to combat MDR (Rangel-Vega et al., 2015; Mookherjee et al., 2018). AHL mediated quorum sensing inhibition was reported to be effective in many pathogens including C.violaceum, Pseudomonas aeruginosa (Kim et al., 2015; Deryabin and Inchagova, 2018; Pérez-López et al., 2018; Soukarieh et al., 2018; Zhou et al., 2018). Since, natural products are the alluring sources of drug discovery, we intended to screen the CviR inhibitors from phytochemicals.

Virtual screening results suggested that numerous chemical moieties were able to interact with the CviR. Based on the GScore and the ability to form H-Bonds, four natural products have been chosen for further studies. Our in silico data revealed that molecular docking is in consistent with Crystallographic structures and in coherence with previous report (Kimyon et al., 2016). Generally, LuxR-type proteins are homodimers and each monomer consists of two domains, a ligand-binding domain (LBD) and a DNA-binding domain (DBD). Upon reception of cognate signal via LBD, they will undergo certain conformational changes, thereby allowing gene expression (Chen et al., 2011). All the QSIs SPL, BN1, BN2, C7X have better GScore than that of $\mathrm{C}_{6} \mathrm{HSL}(-7.052)$. SPL and BN1 have a very similar pattern of 

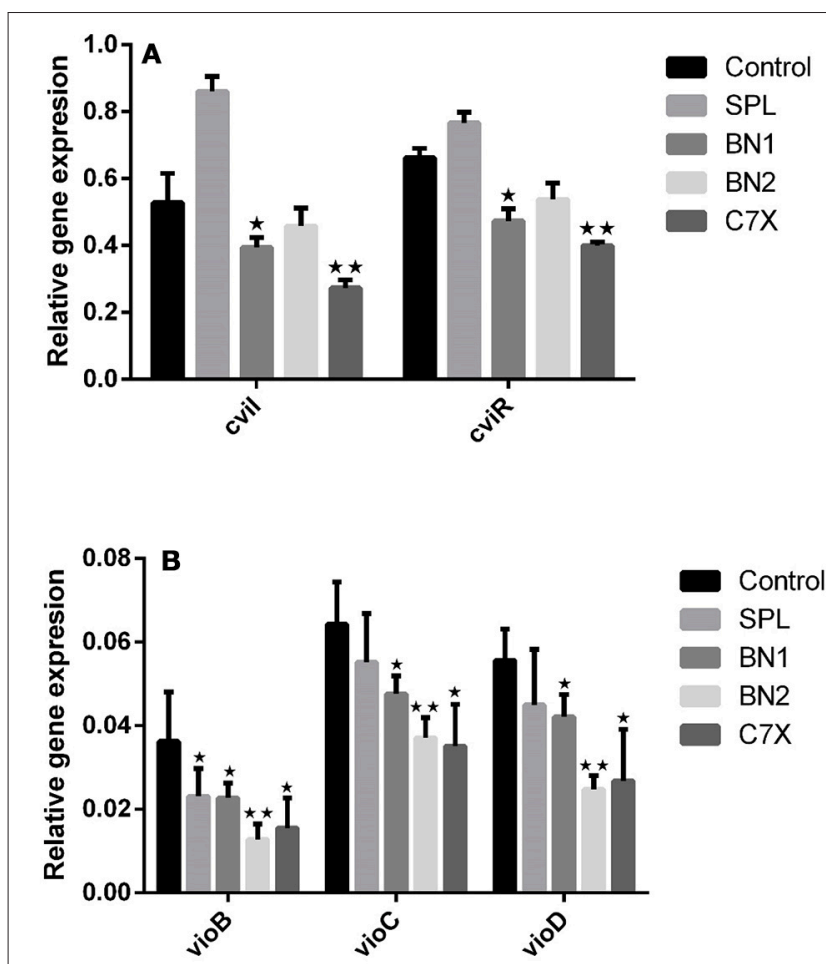

FIGURE 6 | The influence of QSIs on the genes which are under the control of Cvil/CviR mediated quorum sensing. (A) The cvil and cviR genes were significantly suppressed by BN1 and C7X. (B) The genes involved in violacein biosynthesis were significantly suppressed by BN2. ${ }^{*} P<0.05$ and ${ }^{\star \star} P<0.01$.

interaction alike $\mathrm{C}_{6} \mathrm{HSL}$ along with $\mathrm{H}$-bond Met 135. BN2 has a single $\mathrm{H}$-bond with $\operatorname{Trp} 84$, one of the key residue in the binding pocket of $\mathrm{C}_{6} \mathrm{HSL}$ and it is observed to have two pi-pi interactions as well (Figures 1D, 2E). It is speculated that, BN1 and BN2 may induce a closed conformation of CviR, hence it cannot interact with the DNA and thus inhibiting the QS as similar as chlorolactone $(\mathrm{CL})$. It is demonstrated that CL potentially inhibits the C.violaceum QS by interacting with Trp 84 and Asp 97 along with a pi-pi stacking with Tyr 88 which is very similar to the pattern of interaction of BN1 (Swem et al., 2009). Whereas C7X has entirely different pattern of interaction with $4 \mathrm{H}$ bonds and surprisingly, C7X has interaction with Arg 74, which was reported to be present in DBT. Hence, it is hypothesized C7X could inhibit the QS by occupying the $\mathrm{DBD}$ and inducing a closed conformation.

Our findings showed that the AHLs ( $\mathrm{C}_{6} \mathrm{HSL}$ and 3-oxo$\mathrm{C}_{12} \mathrm{HSL}$ ) have four crucial point of interactions such as lactone carbonyl group which forms an $\mathrm{H}$-bond with Trp84 residue, the acyl group amine forms a H-bond with Asp 97 and the carbonyl oxygen which forms $\mathrm{H}$-bonds with Tyr80 and Ser155 which coheres with the results of Ahmed et al. (2013) (Figures 3A-C). Further, it is found that Ser $155_{\mathrm{CviR}}$ and Ser $129_{\text {LasR }}$ were in a distance less than $1 \AA(0.75 \AA)$ and this suggested that Ser in the LBD must be a very essential point of interaction. Even though docking relies on many approximations, lead optimization was often in concert with evaluations and moreover this virtual screening approach saves time, manpower and cost when compared to the traditional approaches.

In AHL mediated QSIs identification process via virtual screening, biofilm formation and violacein quantification assays are the basic and crucial steps. Since violacein pigment is under the control of QS mechanism, C.violacum is considered to be one of the best and easily accessible biomonitor strains to screen QSIs of any origin. Our in vitro studies demonstrated that the QSIs have a potential influence on QS regulated phenotypes at the tested concentrations $(1,10,100 \mu \mathrm{M})$ without affecting the growth (Figures 4A,B and Figure S1). Numerous studies have been reported that the plant-based natural products, such as Vanillin, Naringin, Naringenin, Quercetin, Ellagic acid and Curcumin, reduced the biofilm formation without affecting the growth (Bouyahya et al., 2017). Curcumin was reported to reduce the biofilm and virulence related traits in various uropathogens in a concentration dependent manner (Packiavathy et al., 2014). Carvacrol significantly reduced the biofilm formation $(0.1-0.3 \mathrm{mM})$ of C.violaceum and other pathogens (Burt et al., 2014). Quercetin and quercetin-3-Oarabinoside inhibited violacein production in C. violaceum, at 50 and $100 \mu \mathrm{g} / \mathrm{mL}$, respectively (Vasavi et al., 2014). Isoprenyl caffeate, from manuka propolis found to reduce violacein in agar diffusion assays (Gemiarto et al., 2015). Studies revealed that tannin rich fractions of Terminalia catappa inhibited violacein production (50\%) at $62.5 \mu \mathrm{g}$ per $\mathrm{mL}$ without significantly affecting growth (Taganna et al., 2011). It is observed that our QSIs, have suppressed the QS at minimal concentrations when compared to most of the earlier reports. To demonstrate the effect of QSIs on biofilm, CLSM studies have been performed. Results revealed that the QSIs, BN1 and BN2 have drastically reduced the biofilm (Figures 5D,E), which is in consistent with in vitro biofilm assay. Unfortunately, SPL increased the biofilm formation which is comparable to the control. Though many reports available on biofilm and violacein inhibition of various plant extracts in search of QSIs, the active principle responsible for such effects have not been investigated further in most of the cases. Many synthetic chemicals have also been explored for QSI activity but still they are not taken for further studies.

To investigate the efficacy of QSIs on the genes which are under the control of QS mechanism, qRT-PCR experiment was conducted. The cviI gene which produces the $\mathrm{C}_{6} \mathrm{HSL}$ and the gene $c v i R$ which produces CviR protein were taken into consideration. Data suggest that significant suppression by $\mathrm{BN} 1$ and $\mathrm{C7X}$ is in consistent with the in vitro study results. Our results are in agreement with (Burt et al., 2014) which was observed that $0.3 \mathrm{mM}$ of carvacrol inhibited the cviI gene expression. Hence, these results indicated that QSIs inhibited the production of AHL at gene expression level.

In violacein biosynthetic pathway, the vioB produces a polyketide synthase, which is very essential for biosynthesis of violacein, as it catalyzes the condensation of two tryptophan derivatives. Whereas vioD, vioC are nucleotide-dependent monooxygenases. vioD seems to catalyze the hydroxylation of one tryptophan moiety, whereas VioA seems to catalyze an oxidative deamination in the second tryptophan moiety, and vioC catalyzes intermediate violacein oxidation (August 


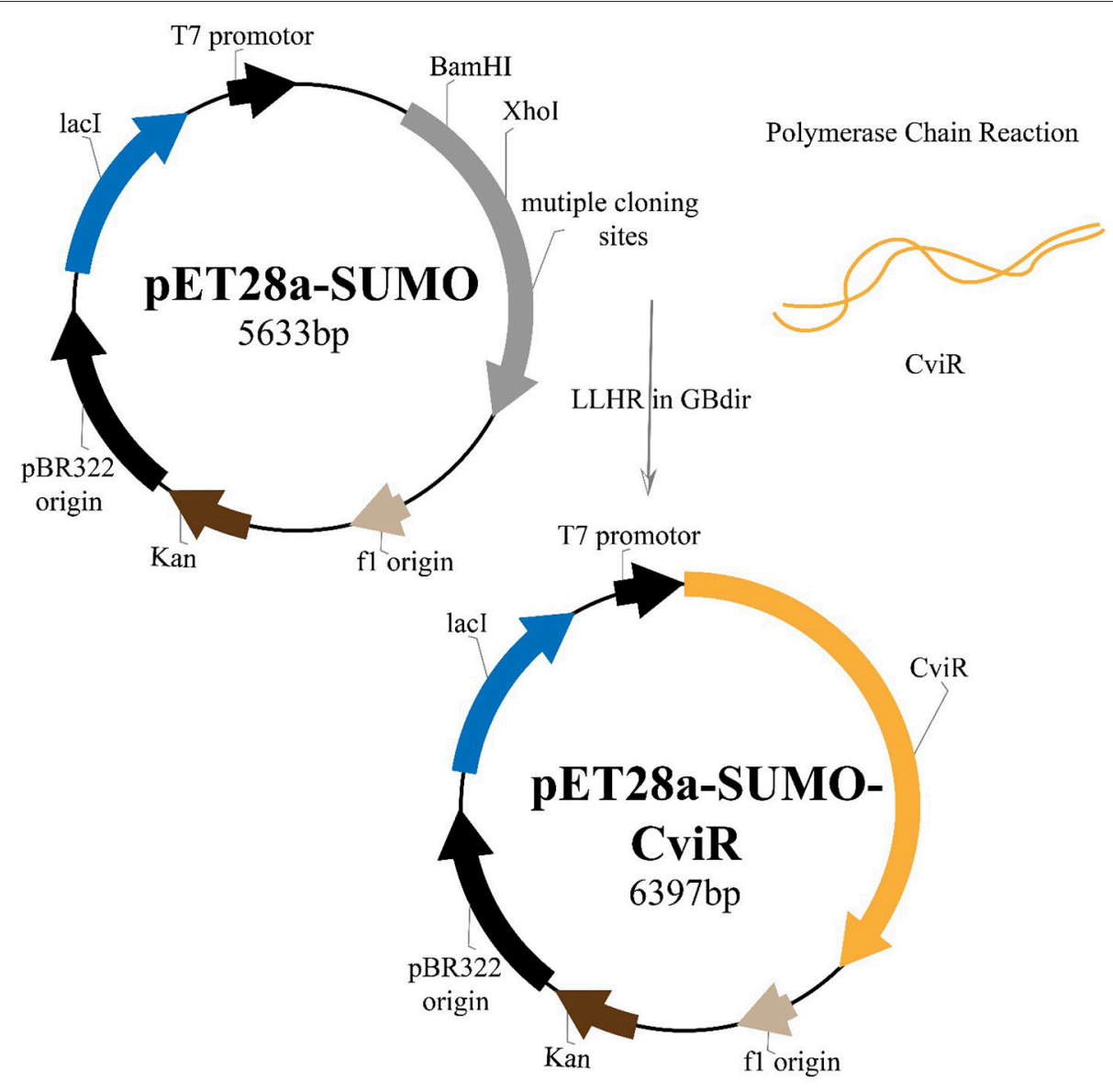

FIGURE 7 | Plasmid construction and the recombineering strategy followed for the heterologous expression of CviR protein.

et al., 2000). To study the effect of these QSIs on genes involved in violacein biosynthetic pathway, we have tested the gene expression of vioB, vioC, and vioD. All the QSIs have significantly suppressed the genes tested. BN1 significantly reduced the expression of $v i o B, v i o C$, and $v i o D$ (Figures 6A,B). It is documented that Manuka propolis PF5 treatment $(300 \mu \mathrm{g} / \mathrm{ml})$ down-regulated $v i o D$, and the key residue was found to be isoprenyl caffeate (Gemiarto et al., 2015). Gene expression study showed the efficacy of QSIs in down regulating the QS related genes which play roles in biofilm formation and virulence directly or indirectly.

Further, to study the molecular interactions between these QSIs and CviR, CviR protein was expressed, isolated and purified for microscale thermophoresis (MST) analysis. For the CviR protein expression, RecET from Rac prophage mediated linear-linear homologous recombination (LLHR) method was followed as per our previous report, which can be used to clone large DNA regions directly from genomic DNA into expression vectors (Wang et al., 2016). MST is a powerful technique to measure biomolecular interactions which based on thermophoresis, the movement of molecules in a temperature gradient. This technique was reported to be highly sensitive that allows precise quantification of molecular interactions (Jerabek-Willemsen et al., 2014). MST results suggest that all the QSIs have potential molecular interaction with purified CviR (Figures 8A-C). The dissociation constant $\left(K_{\mathrm{d}}\right)$ of the BN2 is $45.07 \pm 1.90 \mathrm{~nm}$ (Figure 8C). These data suggest that BNI having a very similar interaction pattern to that of $\mathrm{C}_{6} \mathrm{HSL}$ along with 2 pi-pi interactions, shows very significant interaction with CviR. According to Seidel et al. (2013), the fitting curve may be either S-shaper or mirror S-shaped. The reversal sign of MST amplitude (change in normalized fluorescence) depends on the chemistry of the compound that is titrated (e.g., Charge), its binding site and the conformational change induced upon binding. SPL and BN2 have negative slope suggesting interaction that don't alter the conformation significantly. Whereas the BN1 shows a positive slope suggesting a strong conformational change induced upon complex formation. Probably two pi-pi interactions play a major role in conformational change. Though C7X was not able to fit into the CviR binding pocket, we speculate that it might interfere the QS mechanism by negatively influencing the conformational changes required for the QS activation by interacting with the region near DNA binding domain (DBD) of CviR.

Overall results suggest that except SPL, BN1, BN2, and C7X significantly suppressed the QS of C. violaceum. Sappanol (SPL) is a 3, 4-dihydroxyhomoisoflavan, can be found in Caesalpinia sappan. Butein (BN1) is a chalcone, can be found in Toxicodendron vernicifluum. Bavachin (BN2) is a flavonoid, 

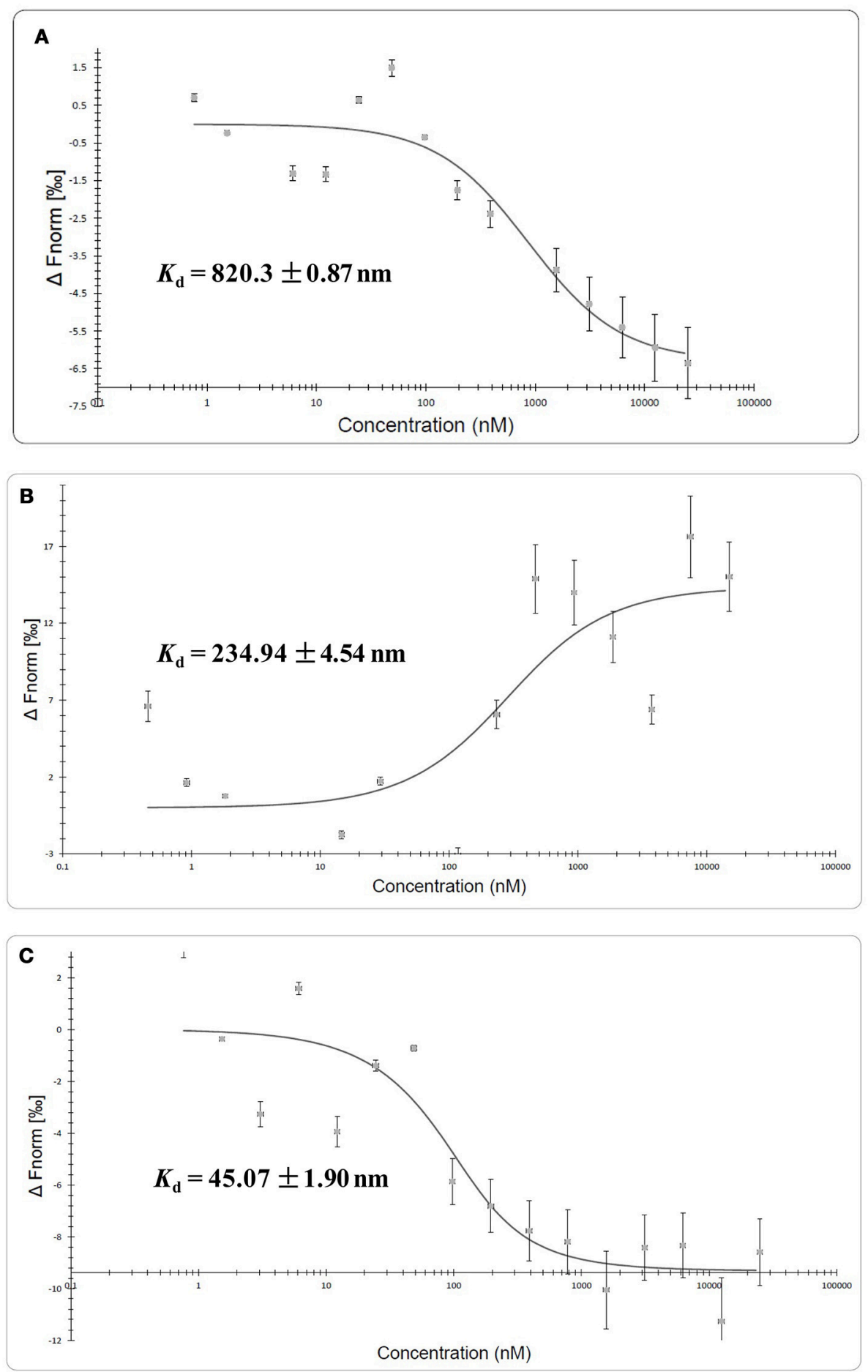

FIGURE 8 | Molecular interaction of QSIs using microscale thermophoresis analysis. (A) Molecular interaction of SPL (B) molecular interaction BN1 (C) molecular interaction BN2. Unfortunately we could not find any such interaction with $\mathrm{C} 7 \mathrm{X}$.

can be found in Psoralea corylifolia. Catechin-7-Xyloside (C7X) is flavan-3-ols, can be found in Spiraea hypericifolia L. All the natural products have their own biological activity profile. Virtual screening, in vitro studies, CLSM analysis of biofilm, qRT-PCR studies and molecular interaction studies using MST, suggest that BN1, BN2 significantly inhibited the CviR-mediated QS, whereas C7X might have a different mode of action and has to be explored further. Though QSIs are potential alternative to antibiotics in 
the battle against MDR pathogens, it is essential to have an eye on the chances for QSIs getting resistance (Kalia et al., 2014; García-Contreras, 2016).

\section{CONCLUSION}

To summarize, our present data from virtual screening, docking analysis, qRT-PCR and MST measurement proved that the phytochemicals BN1, BN2, C7X inhibit CviR-mediated quorum against C.violaceum and represent potential CviR-mediated quorum sensing inhibitors against C.violaceum. Since LuxR homologs are present in more than 100 gram negative pathogens, these QSIs may be developed as a broad spectrum anti-infective drug candidates. Considering the emergence of multi drug resistant pathogens, it is very essential to develop novel drug discovery strategies to find potent drugs against these deadly pathogens. Since natural products always play a major role in medicine and human health, virtual screening of natural products against the molecular drug targets will be a productive approach. It is evident that starting with biological evaluation, gene expression studies and molecular interactions using MST, will help us to get an in-depth understanding of the mode of action of these moieties. It is concluded that BN1 and BN2 inhibiting the C.violaceum by interacting with LBD of CviR. In contrast, C7X interacting with $\mathrm{DBD}$ of CviR and show comparatively less inhibition than BN1 and BN2. Finally thus, this approach will help us to find out effective QSI against various pathogens.

\section{REFERENCES}

Adonizio, A. L., Downum, K., Bennett, B. C., and Mathee, K. (2006). Anti-quorum sensing activity of medicinal plants in southern Florida. J. Ethnopharmacol. 105, 427-435. doi: 10.1016/j.jep.2005.11.025

Ahmed, M., Bird, S., Wang, F., and Palombo, E. A. (2013). In silico investigation of lactone and thiolactone inhibitors in bacterial quorum sensing using molecular modeling. Int. J. Chem. 5, 9-26. doi: 10.5539/ijc.v5n4p9

August, P. R., Grossman, T. H., Minor, C., Draper, M. P., MacNeil, I. A., Pemberton, J. M., et.al. (2000). Sequence analysis and functional characterization of the violacein biosynthetic pathway from Chromobacterium violaceum. J. Mol. Microbiol. Biotechnol. 2, 513-519.

Bassler, B. L. (2002). Small talk: cell-to-cell communication in bacteria. Cell. 109, 421-424. doi: 10.1016/S0092-8674(02)00749-3

Blosser, R. S., and Gray, K. M. (2000). Extraction of violacein from Chromobacterium violaceum provides a new quantitative bioassay for N-acyl homoserine lactone autoinducers. J. Microbiol. Method. 40, 47-55. doi: 10.1016/S0167-7012(99)00136-0

Bouyahya, A., Dakka, N., Et-Touys, A., Abrini, J., and Bakri, Y. (2017). Medicinal plant products targeting quorum sensing for combating bacterial infections. Asian Pac. J. Trop. Med. 10, 729-743. doi: 10.1016/j.apjtm.2017.07.021

Brackman, G., and Coenye, T. (2015). Quorum sensing inhibitors as anti-biofilm agents. Curr. Pharm. Design. 21, 5-11. doi: 10.2174/1381612820666140905114627

Burt, S. A., Ojo-Fakunle, V. T., Woertman, J., and Veldhuizen, E. J. (2014). The natural antimicrobial carvacrol inhibits quorum sensing in Chromobacterium violaceum and reduces bacterial biofilm formation at sub-lethal concentrations. PLoS ONE 9:e93414. doi: 10.1371/journal.pone.0093414

Butler, M. S. (2008). Natural products to drugs: natural product-derived compounds in clinical trials. Nat. Prod. Rep. 25, 475-516. doi: 10.1039/b514294f

\section{AUTHOR CONTRIBUTIONS}

YZ and VR conceived the idea and planned the experiments. VR, LZ and GY performed the experiment. YZ, VR, HW and $\mathrm{AL}$ contributed in data interpretation, $\mathrm{AL}, \mathrm{YZ}$ and VR wrote the manuscript.

\section{FUNDING}

VR received China post-doctoral grant (2015M582103) from China Postdoctoral Science Foundation.

\section{ACKNOWLEDGMENTS}

VR was supported by China post-doctoral grant (2015M582103). We would like to thank Prof. Wei Qian, State Key Laboratory of Plant Genomics, Institute of Microbiology, Chinese Academy of Sciences, Beijing, China for providing the MST facility. We also thank the support from National Natural Science Foundation of China (31670097) and Key Research and Development Program of Shandong Province (2015 GSF12101).

\section{SUPPLEMENTARY MATERIAL}

The Supplementary Material for this article can be found online at: https://www.frontiersin.org/articles/10.3389/fcimb. 2018.00292/full\#supplementary-material

Cai, Z., Yuan, Z. H., Zhang, H., Pan, Y., Wu, Y., Tian, X. Q., et.al. (2017). Fatty acid DSF binds and allosterically activates histidine kinase RpfC of phytopathogenic bacterium Xanthomonas campestris $p v$. campestris to regulate quorum-sensing and virulence. PLoS. Pathog. 13:e1006304. doi: 10.1371/journal.ppat.1006304

Chen, G., Swem, L. R., Swem, D. L., Stauff, D. L., O'Loughlin, C. T.,Jeffrey, P. D., et.al. (2011). A strategy for antagonizing quorum sensing. Mol. Cell. 42, 199-209. doi: 10.1016/j.molcel.2011.04.003

Coughlan, L. M., Cotter, P. D., Hill, C., and Alvarez-Ordóñez, A. (2016). New weapons to fight old enemies: novel strategies for the (bio) control of bacterial biofilms in the food industry. Front. Microbiol. 7:1641. doi: 10.3389/fmicb.2016.01641

Defoirdt, T., Boon, N., and Bossier, P. (2010). Can bacteria evolve resistance to quorum sensing disruption?. PLoS Pathog. 6:e1000989. doi: 10.1371/journal.ppat.1000989

Delago, A., Mandabi, A., and Meijler, M. M. (2016). Natural quorum sensing inhibitors-small molecules, big messages. Isr. J. Chem. 56, 310-320. doi: 10.1002/ijch.201500052

Deryabin, D. G., and Inchagova, K. S. (2018). Inhibitory effect of aminoglycosides and tetracyclines on quorum sensing in Chromobacterium violaceum. Microbiology 87, 1-8. doi: 10.1134/S002626171801006X

Fantinatti-Garboggini, F., Almeida, R. D., Portillo Vdo, A., Barbosa, T. A., Trevilato, P. B., Neto, C. E. R., et.al. (2004). Drug resistance in Chromobacterium violaceum. Genet. Mol. Res. 3, 134-147.

Ferri, M., Ranucci, E., Romagnoli, P., and Giaccone, V. (2017). Antimicrobial resistance: a global emerging threat to public health systems. Crit. Rev. Food. Sci. 57, 2857-2876. doi: 10.1080/10408398.2015.1077192

Friesner, R. A., Murphy, R. B., Repasky, M. P., Frye, L. L., Greenwood, J. R., Halgren, T. A., et.al. (2006). Extra precision glide: docking and scoring incorporating a model of hydrophobic enclosure for protein-ligand complexes. J. Med. Chem. 49, 6177-6196. doi: 10.1021/jm051256o 
García-Contreras, R. (2016). Is quorum sensing interference a viable alternative to treat Pseudomonas aeruginosa infections? Front. Microbiol. 7:1454. doi: $10.3389 /$ fmicb.2016.01454

Gemiarto, A. T., Ninyio, N. N., Lee, S. W., Logis, J., Fatima, A., Chan, E. W., et.al. (2015). Isoprenyl caffeate, a major compound in manuka propolis, is a quorumsensing inhibitor in Chromobacterium violaceum. A. Van. Leeuw. J. Microb. 108, 491-504. doi: 10.1007/s10482-015-0503-6

Harvey, A. L. (2008). Natural products in drug discovery. Drug Discov. Today 13, 894-901. doi: 10.1016/j.drudis.2008.07.004

Jerabek-Willemsen, M., André, T., Wanner, R., Roth, H. M., Duhr, S., Baaske, P., et.al. (2014). MicroScale thermophoresis: interaction analysis and beyond. J. Mol. Struct. 1077, 101-113. doi: 10.1016/j.molstruc.2014.03.009

Justo, G. Z., and Durán, N. (2017). Action and function of Chromobacterium violaceum in health and disease. Best. Pract. Res. Cl. Ga. 31, 649-656. doi: 10.1016/j.bpg.2017.10.002

Kalia, V. C. (2013). Quorum sensing inhibitors: an overview. Biotechnol. Adv. 31, 224-245. doi: 10.1016/j.biotechadv.2012.10.004

Kalia, V. C. (2015). Quorum sensing vs quorum quenching: a battle with no end in sight. New Delhi: Springer.

Kalia, V. C., Wood, T. K., and Kumar, P. (2014). Evolution of resistance to quorumsensing inhibitors. Microb. Ecol. 68, 13-23. doi: 10.1007/s00248-013-0316-y

Kim, H. S., Lee, S. H., Byun, Y., and Park, H. D. (2015). 6-Gingerol reduces Pseudomonas aeruginosa biofilm formation and virulence via quorum sensing inhibition. Sci. Rep. 5:8656. doi: 10.1038/srep08656

Kimyon, Ö., Ulutürk, Z. I., Nizalapur, S., Lee, M., Kutty, S. K., Beckmann, S., et.al. (2016). N-Acetylglucosamine inhibits LuxR, LasR and CviR based quorum sensing regulated gene expression levels. Front. Microbiol. 7:1313. doi: $10.3389 /$ fmicb. 2016.01313

Laxminarayan, R., Matsoso, P., Pant, S., Brower, C., Røttingen, J. A., Klugman, K., et.al. (2016). Access to effective antimicrobials: a worldwide challenge. Lancet. 387, 168-175. doi: 10.1016/S0140-6736(15)00474-2

McClean, K. H., Winson, M. K., Fish, L., Taylor, A., Chhabra, S. R., Camara, M., et.al. (1997). Quorum sensing and Chromobacterium violaceum: exploitation of violacein production and inhibition for the detection of $\mathrm{N}$-acylhomoserine lactones. Microbiology. 143, 3703-3711. doi: 10.1099/00221287-143-12-3703

Miller, M. B., and Bassler, B. L. (2001). Quorum sensing in bacteria. Annu. Rev. Microbiol. 55, 165-199. doi: 10.1146/annurev.micro.55.1.165

Mookherjee, A., Singh, S., and Maiti, M. K. (2018). Quorum sensing inhibitors: can endophytes be prospective sources? Arch. Microbiol. 200, 355-369. doi: 10.1007/s00203-017-1437-3

Ni, N., Choudhary, G., Li, M., and Wang, B. (2008). Pyrogallol and its analogs can antagonize bacterial quorum sensing in Vibrio harveyi. Bioorg. Med. Chem. Lett. 18, 1567-1572. doi: 10.1016/j.bmcl.2008.01.081

O'toole, G. A., and Kolter, R. (1998). Flagellar and twitching motility are necessary for Pseudomonas aeruginosa biofilm development. Mol. Microbiol. 30, 295-304. doi: 10.1046/j.1365-2958.1998.01062.x

Packiavathy, I. A., Priya, S., Pandian, S. K., and Ravi, A. V. (2014). Inhibition of biofilm development of uropathogens by curcumin-an antiquorum sensing agent from Curcuma longa. Food Chem. 148, 453-460. doi: 10.1016/j.foodchem.2012.08.002

Paul, D., Gopal, J., Kumar, M., and Manikandan, M. (2017). Nature to the natural rescue: silencing microbial chats. Chem-Biol. Interact. 280, 86-98. doi: 10.1016/j.cbi.2017.12.018

Pérez-López, M., García-Contreras, R., Soto-Hernández, M., Rodríguez-Zavala, J. S., Martínez-Vázquez, M., Prado-Galbarro, F. J., et.al. (2018). Antiquorum sensing activity of seed oils from oleaginous plants and protective effect during challenge with Chromobacterium violaceum. J. Med. Food. 21, 356-363. doi: 10.1089/jmf.2017.0080

Rangel-Vega, A., Bernstein, L. R., Mandujano Tinoco, E. A., García-Contreras, S. J., and García-Contreras, R. (2015). Drug repurposing as an alternative for the treatment of recalcitrant bacterial infections. Front. Microbiol. 6:282. doi: $10.3389 /$ fmicb.2015.00282

Rasmussen, T. B., and Givskov, M. (2006). Quorum sensing inhibitors: a bargain of effects. Microbiology. 152, 895-904. doi: 10.1099/mic.0.28601-0

Ren, D., Zuo, R., González Barrios, A. F., Bedzyk, L. A., Eldridge, G. R., Pasmore, M. E., et al. (2005). Differential gene expression for investigation of Escherichia coli biofilm inhibition by plant extract ursolic acid. Appl. Environ. Microbiol. 71, 4022-4034. doi: 10.1128/AEM.71.7.4022-4034.2005

Seidel, S. A., Dijkman, P. M., Lea, W. A., van den Bogaart, G., Jerabek-Willemsen, M., Lazic, A., et al. (2013). Microscale thermophoresis quantifies biomolecular interactions under previously challenging conditions. Methods 59, 301-315. doi: 10.1016/j.ymeth.2012.12.005

Soukarieh, F., Vico Oton, E., Dubern, J. F., Gomes, J., Halliday, N., de Pilar Crespo, M., et.al. (2018). In silico and in vitro-guided identification of inhibitors of alkylquinolone-dependent quorum sensing in Pseudomonas aeruginosa. Molecules. 23:257. doi: 10.3390/molecules23020257

Stauff, D. L., and Bassler, B. L. (2011). Quorum sensing in Chromobacterium violaceum: DNA recognition and gene regulation by the CviR receptor. J. Bacteriol. 193, 3871-3878. doi: 10.1128/JB.05125-11

Swem, L. R., Swem, D. L., O'Loughlin, C. T., Gatmaitan, R., Zhao, B., Ulrich, S. M., et.al. (2009). A quorum-sensing antagonist targets both membrane-bound and cytoplasmic receptors and controls bacterial pathogenicity. Mol. Cell. 35, 143-153. doi: 10.1016/j.molcel.2009.05.029

Taganna, J. C., Quanico, J. P., Perono, R. M. G., Amor, E. C., and Rivera, W. L. (2011). Tannin-rich fraction from Terminalia catappa inhibits quorum sensing (QS) in Chromobacterium violaceum and the QS-controlled biofilm maturation and LasA staphylolytic activity in Pseudomonas aeruginosa. J. Ethnopharmacol. 134, 865-871. doi: 10.1016/j.jep.2011.01.028

Tornimbene, B., Eremin, S., Escher, M., Griskeviciene, J., Manglani, S., and Pessoa-Silva, C. L. (2018). WHO global antimicrobial resistance surveillance system early implementation 2016-17. Lancet Infect. Dis. 18, 241-242. doi: 10.1016/S1473-3099(18)30060-4

Uroz, S., Dessaux, Y., and Oger, P. (2009). Quorum sensing and quorum quenching: the yin and yang of bacterial communication. Chem. Bio. Chem. 10, 205-216. doi: 10.1002/cbic.200800521

Vasavi, H. S., Arun, A. B., and Rekha, P. D. (2014). Anti-quorum sensing activity of Psidium guajava L. flavonoids against Chromobacterium violaceum and Pseudomonas aeruginosa PAO1. Microbiol. Immunol. 58, 286-293. doi: 10.1111/1348-0421.12150

Vattem, D. A., Mihalik, K., Crixell, S. H., and McLean, R. J. C. (2007). Dietary phytochemicals as quorum sensing inhibitors. Fitoterapia. 78, 302-310. doi: 10.1016/j.fitote.2007.03.009

Wagner, S., Sommer, R., Hinsberger, S., Lu, C., Hartmann, R. W., Empting, M., et.al. (2016). Novel strategies for the treatment of Pseudomonas aeruginosa infections. J. Med. Chem. 59, 5929-5969. doi: 10.1021/acs.jmedchem.5b01698

Wang, H., Li, Z., Jia, R., Hou, Y., Yin, J., Bian, X., et al. (2016). RecET direct cloning and Red $\alpha \beta$ recombineering of biosynthetic gene clusters, large operons or single genes for heterologous expression. Nat. Protoc. 11, 1175-1190. doi: $10.1038 /$ nprot.2016.054

Williams, P. (2017). Strategies for inhibiting quorum sensing. Emerg. Top. Life Sci. 1, 23-30. doi: 10.1042/ETLS20160021

Zhao, T., and Liu, Y. (2010). N-acetylcysteine inhibit biofilms produced by Pseudomonas aeruginosa. BMC Microbiol. 10:140. doi: 10.1186/1471-2180-10-140

Zhou, J. W., Luo, H. Z., Jiang, H., Jian, T. K., Chen, Z. Q., and Jia, A. Q. (2018). Hordenine, a novel quorum sensing inhibitor and anti-biofilm agent against Pseudomonas aeruginosa. J. Agr. Food. Chem. 66, 1620-1628. doi: 10.1021/acs.jafc.7b05035

Conflict of Interest Statement: The authors declare that the research was conducted in the absence of any commercial or financial relationships that could be construed as a potential conflict of interest.

The reviewer YGT and handling Editor declared their shared affiliation.

Copyright (c) 2018 Ravichandran, Zhong, Wang, Yu, Zhang and Li. This is an openaccess article distributed under the terms of the Creative Commons Attribution License (CC BY). The use, distribution or reproduction in other forums is permitted, provided the original author(s) and the copyright owner(s) are credited and that the original publication in this journal is cited, in accordance with accepted academic practice. No use, distribution or reproduction is permitted which does not comply with these terms. 